Supporting Information for

\title{
A Facile Synthetic Method to Prepare Fluorescently Labeled ROMP Polymers
}

\author{
Kenny S. Roberts and Nicole S. Sampson*
}

\section{Materials and Methods}

Amino acids and coupling agents used were purchased from Advanced Chem Tech. (Louisville, KY) or PerSeptive Biosystems (Framingham, MA). Solvents were obtained from Fisher Scientific Inc (Springfield, NJ), and Aldrich (Milwaukee, WI).

$\mathrm{Cl}_{2}\left(\mathrm{PCy}_{3}\right)\left(\mathrm{H}_{2} \mathrm{IMes}\right) \mathrm{Ru}=\mathrm{CHPh}$ was purchased from Fluka (Milwaukee, WI). Oregon Green 488 cadaverine was purchased from Molecular Probes (Eugene, OR). $\mathrm{CH}_{2} \mathrm{Cl}_{2}$ was freshly distilled from $\mathrm{CaH} ; \mathrm{CF}_{3} \mathrm{CH}_{2} \mathrm{OH}, \mathrm{CH}_{3} \mathrm{OH}$, and $\mathrm{Et}_{2} \mathrm{O}$, were used without further purification. All reactions were carried out under an $\mathrm{N}_{2}$ or Ar atmosphere in oven-dried glassware. Moisture and oxygen-sensitive reagents were handled in an $\mathrm{N}_{2}$-filled drybox. 5-Norbornene-exo-carboxylic acid was synthesized according to the literature. ${ }^{1}$

Analytical thin layer chromatography (TLC) was performed on precoated silica gel plates $\left(60 \mathrm{~F}_{254}\right)$, and flash chromatography on silica gel-60 (230-400 mesh). TLC spots were detected by UV light and by staining with phosphomolybdic acid (PMA). Peptides were purified by flash column chromatography on silica gel-60. Purity of all peptide monomers were assessed by RP-HPLC using a Vydac $\mathrm{C}_{18}$. Gradient elution was performed at $1 \mathrm{~mL} / \mathrm{min}$ with acetonitrile and water (both containing TFA, $0.1 \%$ ). Bruker AC-250, Gemini 2300, Inova500, and Inova600 MHz NMR spectrometers were used to

\footnotetext{
${ }^{1}$ Manning, D.D.; Strong, L.E.; Hu, X.; Beck, P.J.; Kiessling, L.L. Tetrahedron 1997, 53, 11937.
} 
perform NMR analysis, and spectra were recorded in $\mathrm{CDCl}_{3}$ unless otherwise noted. Chemical shifts are quoted in parts per million (ppm) and ${ }^{1} \mathrm{H}$ NMR data are assumed to be first order.

\section{Peptide synthesis}

General procedure for amino acid coupling. A typical amino acid coupling was carried out in dry $\mathrm{CH}_{2} \mathrm{Cl}_{2}$ with TBTU/HOBt (1.1 eq / $\left.0.37 \mathrm{eq}\right)$ and DIEA (1.6 eq). Each reaction was carried out under Ar at a final concentration of $0.7 \mathrm{M}$ in the amine compound, and a 1.1-fold excess of the carboxylic acid component. Upon completion of the reaction, the mixture was diluted with $\mathrm{CH}_{2} \mathrm{Cl}_{2}$, washed with $1 \mathrm{~N} \mathrm{HCl}$ and $5 \%$ $\mathrm{NaHCO}_{3}$, and the organic layer was dried with $\mathrm{Na}_{2} \mathrm{SO}_{4}$. The solvent was rotary evaporated. The peptide was purified by flash chromatography eluting with $10 \%$ acetone $/ \mathrm{CH}_{2} \mathrm{Cl}_{2}$ or $20 \% \mathrm{EtOAc} / \mathrm{CH}_{2} \mathrm{Cl}_{2}$.

General procedure for Cbz hydrogenation. A methanolic solution of Cbz-protected peptide $(0.3 \mathrm{M})$ and $10 \% \mathrm{Pd}-\mathrm{C}(0.05 \mathrm{eq})$ was stirred under an $\mathrm{H}_{2}$ atmosphere for $2 \mathrm{~h}$. The catalyst was removed by filtration and the amine used without further purification.

General procedure for Fmoc removal. A solution of Fmoc-protected peptide in dry $\mathrm{CH}_{2} \mathrm{Cl}_{2}(0.5 \mathrm{M})$, was treated with octanethiol ( $\left.0.1 \mathrm{eq}\right)$ and a catalytic amount of DBU (0.001 eq). The reaction was stirred at RT for $16 \mathrm{~h}$. The solvent was concentrated and the product was purified by flash column chromatography eluting with a step gradient of ranging from $2 \%$ to $50 \% \mathrm{EtOAc} / \mathrm{CH}_{2} \mathrm{Cl}_{2}$ 


\section{Peptide 3a}

Z-VT(tBu)-OMe. H-T(tBu)-OMe and Z-V-OH were coupled and purified to yield $1.26 \mathrm{~g}(97 \%)$ of Z-VT(tBu)-OMe. ${ }^{1} \mathrm{H}-\mathrm{NMR}(250 \mathrm{MHz}): \delta 7.36(\mathrm{~m}, 5 \mathrm{H}), 6.42(\mathrm{~d}, \mathrm{~J}=$ 10.0, 1H), $5.49(\mathrm{~d}, \mathrm{~J}=7.5,1 \mathrm{H}), 5.11(\mathrm{~s}, 2 \mathrm{H}), 4.47(\mathrm{dd}, \mathrm{J}=8.8$ and $1.3,1 \mathrm{H}), 4.25(\mathrm{~m}, 1 \mathrm{H})$, $4.22(\mathrm{~m}, 1 \mathrm{H}), 3.69(\mathrm{~s}, 3 \mathrm{H}), 2.12(\mathrm{~m}, 1 \mathrm{H}), 1.15(\mathrm{~d}, \mathrm{~J}=5.0,3 \mathrm{H}), 1.10(\mathrm{~s}, 9 \mathrm{H}), 1.02(\mathrm{~d}, \mathrm{~J}=$ $7.5,3 \mathrm{H}), 0.97(\mathrm{~d}, \mathrm{~J}=5.0,3 \mathrm{H})$.

$Z-D(t B u) V T(t B u)-O M e$. Z-VT(tBu)-OMe was deprotected and coupled to Z-D(tBu)$\mathrm{OH}$ to yield $0.822 \mathrm{~g}(98 \%)$ of $\mathrm{Z}-\mathrm{D}(\mathrm{tBu}) \mathrm{VT}(\mathrm{tBu})-\mathrm{OMe} .{ }^{1} \mathrm{H}-\mathrm{NMR}(250 \mathrm{MHz}): \delta 7.31(\mathrm{~m}$, $5 \mathrm{H}), 7.12(\mathrm{~d}, \mathrm{~J}=10.0,1 \mathrm{H}), 6.40(\mathrm{~d}, \mathrm{~J}=10.0,1 \mathrm{H}), 6.05(\mathrm{~d}, \mathrm{~J}=7.5,1 \mathrm{H}), 5.10(\mathrm{~s}, 2 \mathrm{H}), 4.55$ $(\mathrm{m}, 1 \mathrm{H}), 4.43(\mathrm{dd}, \mathrm{J}=8.8$ and $1.3,1 \mathrm{H}), 4.33(\mathrm{dd}, \mathrm{J}=7.5$ and $5.0,1 \mathrm{H}), 4.20(\mathrm{~m}, 1 \mathrm{H}), 3.68$ $(\mathrm{s}, 3 \mathrm{H}), 2.90(\mathrm{dd}, \mathrm{J}=17.0$ and 5.0, $1 \mathrm{H}), 2.60(\mathrm{dd}, \mathrm{J}=17.5$ and 5.0, $1 \mathrm{H}), 2.12(\mathrm{~m}, 1 \mathrm{H}), 1.40$ $(\mathrm{s}, 9 \mathrm{H}), 1.13(\mathrm{~d}, \mathrm{~J}=7.5,3 \mathrm{H}), 1.10(\mathrm{~s}, 9 \mathrm{H}), 0.96(\mathrm{~d}, \mathrm{~J}=5.0,3 \mathrm{H}), 0.93(\mathrm{~d}, \mathrm{~J}=5.0,3 \mathrm{H})$.

Fmoc-C(Trt)D(tBu)VT(tBu)-OMe. Z-D(tBu)VT(tBu)-OMe was deprotected and coupled to Fmoc-C(Trt)-OH to yield 0.873 g (95\%) of Fmoc-C(Trt)D(tBu)VT(tBu)OMe. ${ }^{1} \mathrm{H}-\mathrm{NMR}(300 \mathrm{MHz}): \delta 7.74(\mathrm{dd}, \mathrm{J}=6.6$ and 6.3, 2H), $7.56(\mathrm{~m}, 2 \mathrm{H}), 7.43(\mathrm{~m}, 7 \mathrm{H})$, $7.38(\mathrm{~m}, 2 \mathrm{H}), 7.25(\mathrm{~m}, 13 \mathrm{H}), 7.13(\mathrm{~d}, \mathrm{~J}=8.7,1 \mathrm{H}), 6.38(\mathrm{~d}, \mathrm{~J}=9.0,1 \mathrm{H}), 4.96(\mathrm{~d}, \mathrm{~J}=6.0$, 1H), $4.69(\mathrm{~m}, 1 \mathrm{H}), 4.46(\mathrm{dd}, \mathrm{J}=8.7$ and 1.3, $1 \mathrm{H}), 4.35(\mathrm{~d}, \mathrm{~J}=6.6,2 \mathrm{H}), 4.22(\mathrm{~m}, 3 \mathrm{H}), 3.69$ (s, 3H), $2.90(\mathrm{dd}, \mathrm{J}=17.1$ and 4.1, 1H), $2.73(\mathrm{~m}, 2 \mathrm{H}), 2.54(\mathrm{dd}, \mathrm{J}=17.3$ and $6.2,1 \mathrm{H})$, $2.00(\mathrm{~m}, 1 \mathrm{H}), 1.39(\mathrm{~s}, 9 \mathrm{H}), 1.14(\mathrm{~d}, \mathrm{~J}=6.3,3 \mathrm{H}), 1.10(\mathrm{~s}, 9 \mathrm{H}), 0.90(\mathrm{~d}, \mathrm{~J}=6.9,3 \mathrm{H}), 0.85(\mathrm{~d}$, $\mathrm{J}=6.6,3 \mathrm{H})$.

Fmoc-E(tBu)C(Trt)D(tBu)VT(tBu)-OMe. Fmoc-C(Trt)D(tBu)VT(tBu)-OMe was deprotected and coupled to Fmoc-E(tBu)-OH to yield $0.660 \mathrm{~g}(95 \%)$ of Fmoc$\mathrm{E}(\mathrm{tBu}) \mathrm{C}(\mathrm{Trt}) \mathrm{D}(\mathrm{tBu}) \mathrm{VT}(\mathrm{tBu})-\mathrm{OMe} .{ }^{1} \mathrm{H}-\mathrm{NMR}(300 \mathrm{MHz}): \delta 7.76(\mathrm{~d}, \mathrm{~J}=6.0,2 \mathrm{H}), 7.59(\mathrm{~m}$, 
2H), $7.40(\mathrm{~m}, 7 \mathrm{H}), 7.28(\mathrm{~m}, 10 \mathrm{H}), 7.14(\mathrm{~m}, 4 \mathrm{H}), 6.53(\mathrm{~d}, \mathrm{~J}=6.0,1 \mathrm{H}), 6.42(\mathrm{~d}, \mathrm{~J}=7.0,1 \mathrm{H})$, $6.03(\mathrm{~d}, \mathrm{~J}=6.3,1 \mathrm{H}), 4.73(\mathrm{~m}, 1 \mathrm{H}), 4.46(\mathrm{dd}, \mathrm{J}=9.3$ and 5.0, 1H), $4.35(\mathrm{~m}, 2 \mathrm{H}), 4.22(\mathrm{~m}$, 1H), $4.13(\mathrm{~m}, 4 \mathrm{H}), 3.91(\mathrm{~m}, 1 \mathrm{H}), 3.68(\mathrm{~s}, 3 \mathrm{H}), 2.81(\mathrm{dd}, \mathrm{J}=17$ and 5.3, $1 \mathrm{H}), 2.62(\mathrm{~m}, 3 \mathrm{H})$, $2.38(\mathrm{~m}, 3 \mathrm{H}), 2.08(\mathrm{~m}, 2 \mathrm{H}), 1.86(\mathrm{~m}, 1 \mathrm{H}), 1.45(\mathrm{~s}, 9 \mathrm{H}), 1.40(\mathrm{~s}, 9 \mathrm{H}), 1.13(\mathrm{~d}, \mathrm{~J}=6.3,3 \mathrm{H})$, $1.10(\mathrm{~s}, 9 \mathrm{H}), 0.92(\mathrm{~d}, \mathrm{~J}=6.6,3 \mathrm{H}), 0.88(\mathrm{~d}, \mathrm{~J}=6.9,3 \mathrm{H})$.

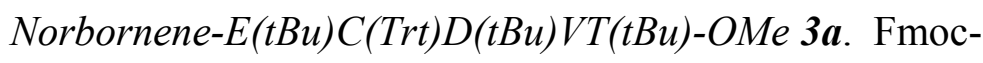
$\mathrm{E}(\mathrm{tBu}) \mathrm{C}(\mathrm{Trt}) \mathrm{D}(\mathrm{tBu}) \mathrm{VT}(\mathrm{tBu})-\mathrm{OMe}$ was deprotected and coupled to 5-norbornene-exocarboxylic acid to yield $0.751 \mathrm{~g}(89 \%)$ of norbornene- $\mathrm{E}(\mathrm{tBu}) \mathrm{C}(\mathrm{Trt}) \mathrm{D}(\mathrm{tBu}) \mathrm{VT}(\mathrm{tBu})-\mathrm{OMe}$, 1. ${ }^{1} \mathrm{H}-\mathrm{NMR}(250 \mathrm{MHz}): \delta 7.38(\mathrm{~m}, 6 \mathrm{H}), 7.20(\mathrm{~m}, 11 \mathrm{H}), 6.98(\mathrm{dd}, \mathrm{J}=8.1$ and $6.3,1 \mathrm{H})$, $6.72(\mathrm{dd}, \mathrm{J}=7.2$ and $3.3,1 \mathrm{H}), 6.47(\mathrm{dd}, \mathrm{J}=9.0$ and $2.7,1 \mathrm{H}), 6.0(\mathrm{~m}, 2 \mathrm{H}), 4.74(\mathrm{~m}, 1 \mathrm{H})$, $4.43(\mathrm{dd}, \mathrm{J}=8.7$ and 1.8, 1H), $4.19(\mathrm{~m}, 3 \mathrm{H}), 3.92(\mathrm{~m}, 1 \mathrm{H}), 3.65(\mathrm{~s}, 3 \mathrm{H}), 2.78(\mathrm{~m}, 5 \mathrm{H})$, $2.49(\mathrm{~m}, 2 \mathrm{H}), 2.27(\mathrm{~m}, 1 \mathrm{H}), 2.02(\mathrm{~m}, 3 \mathrm{H}), 1.83(\mathrm{~m}, 2 \mathrm{H}), 1.60(\mathrm{~m}, 2 \mathrm{H}), 1.41(\mathrm{~d}, \mathrm{~J}=4.5$, 9H), 1.39 (s, 9H), $1.24(\mathrm{~m}, 2 \mathrm{H}), 1.11(\mathrm{~d}, \mathrm{~J}=6.3,3 \mathrm{H}), 1.07$ (s, 9H), $0.90(\mathrm{~d}, \mathrm{~J}=6.6,3 \mathrm{H})$, $0.87(\mathrm{~d}, \mathrm{~J}=6.9,3 \mathrm{H}) .{ }^{13} \mathrm{C}(250 \mathrm{MHz}) \delta 18.04,19.01,20.82,26.41,27.97,28.26,30.44$, $30.54,30.77,32.22,32.96,33.05,36.72,41.48,44.39,46.33,46.98,49.72,51.97,52.51$, $52.61,54.15,57.73,58.86,73.95,81.19,81.31,126.83,128.02,129.45,135.87,138.07$, $144.15,169.33,169.96,170.56,170.76,171.00,171.36,173.66,176.73$. MALDI, calcd for $(\mathrm{MNa})^{+} 1132.89$, found 1133.96 . HPLC purity was $98 \%$.

\section{Peptide 3b}

Z-VD $(t B u)-O M e$. $\mathrm{H}-\mathrm{D}(\mathrm{tBu})-\mathrm{OMe}$ and $\mathrm{Z}-\mathrm{V}-\mathrm{OH}$ were coupled and purified to yield $0.800 \mathrm{~g}(97 \%)$ of Z-VD(tBu)-OMe. ${ }^{1} \mathrm{H}-\mathrm{NMR}(300 \mathrm{MHz}): \delta 7.39(\mathrm{~m}, 5 \mathrm{H}), 6.83(\mathrm{~d}$, $\mathrm{J}=8.7,1 \mathrm{H}), 5.45(\mathrm{~d}, \mathrm{~J}=8.4,1 \mathrm{H}), 5.15(\mathrm{~s}, 2 \mathrm{H}), 4.86(\mathrm{~m}, 1 \mathrm{H}), 4.11(\mathrm{~m}, 1 \mathrm{H}), 3.77(\mathrm{~s}, 3 \mathrm{H})$, 
$3.03(\mathrm{dd}, \mathrm{J}=17.1$ and 4.2, 1H), $2.74(\mathrm{dd}, \mathrm{J}=17.1$ and 4.2, 1H), $2.21(\mathrm{~m}, 1 \mathrm{H}), 1.46(\mathrm{~s}, 9 \mathrm{H})$, $1.04(\mathrm{~d}, \mathrm{~J}=6.6,3 \mathrm{H}), 0.98(\mathrm{~d}, \mathrm{~J}=6.9,3 \mathrm{H})$.

$Z-E(t B u) V D(t B u)-O M e$. Z-VD(tBu)-OMe was deprotected and coupled to Z-E(tBu)OH to yield $0.751 \mathrm{~g}(98 \%)$ of $\mathrm{Z}-\mathrm{E}(\mathrm{tBu}) \mathrm{VD}(\mathrm{tBu})-\mathrm{OMe} .{ }^{1} \mathrm{H}-\mathrm{NMR}(300 \mathrm{MHz}): \delta 7.35(\mathrm{~m}$, 5H), $7.10(\mathrm{~d}, \mathrm{~J}=8.4,1 \mathrm{H}), 7.00(\mathrm{~d}, \mathrm{~J}=8.7,1 \mathrm{H}), 5.87(\mathrm{~d}, \mathrm{~J}=7.5,1 \mathrm{H}), 5.13(\mathrm{~s}, 2 \mathrm{H}), 4.85(\mathrm{~m}$, 1H), $4.35(\mathrm{~m}, 2 \mathrm{H}), 3.76(\mathrm{~s}, 3 \mathrm{H}), 2.96(\mathrm{dd}, \mathrm{J}=17.0$ and 4.8, 1H), 2.73 (dd, J=17.0 and 4.4, 1H), $2.42(\mathrm{~m}, 2 \mathrm{H}), 2.15(\mathrm{~m}, 2 \mathrm{H}), 1.98(\mathrm{~m}, 1 \mathrm{H}), 1.46$ (s, 9H), 1.46 (s, 9H), 0.99 (d, J=6.9, $3 \mathrm{H}), 0.97(\mathrm{~d}, \mathrm{~J}=6.9,3 \mathrm{H})$.

$Z-T(t B u) E(t B u) V D(t B u)-O M e . Z-E(t B u) V D(t B u)-O M e$ was deprotected and coupled to $\mathrm{Z}-\mathrm{T}(\mathrm{tBu})-\mathrm{OH}$ to yield $0.702 \mathrm{~g}(96 \%)$ of $\mathrm{Z}-\mathrm{T}(\mathrm{tBu}) \mathrm{E}(\mathrm{tBu}) \mathrm{VD}(\mathrm{tBu})-\mathrm{OMe} .{ }^{1} \mathrm{H}-\mathrm{NMR}$ $(500 \mathrm{MHz}): \delta 7.76(\mathrm{~d}, \mathrm{~J}=7.5,1 \mathrm{H}), 7.35(\mathrm{~m}, 5 \mathrm{H}), 6.99(\mathrm{~d}, \mathrm{~J}=8.0,1 \mathrm{H}), 6.88(\mathrm{~d}, \mathrm{~J}=8.5,1 \mathrm{H})$, $5.90(\mathrm{~d}, \mathrm{~J}=5.5,1 \mathrm{H}), 5.15(\mathrm{~d}, \mathrm{~J}=12.5,1 \mathrm{H}), 5.10(\mathrm{~d}, \mathrm{~J}=12.0,1 \mathrm{H}), 4.82(\mathrm{dt}, \mathrm{J}=9.0$ and 4.5 , 1H), $4.42(\mathrm{dd}, \mathrm{J}=13.3$ and 7.3, 1H), $4.28(\mathrm{dd}, \mathrm{J}=8.3$ and 5.8, 1H), 4.19 (bs, 2H), 3.75 (s, 3H), $2.93(\mathrm{dd}, \mathrm{J}=16.8$ and 4.8, 1H), $2.72(\mathrm{dd}, \mathrm{J}=17.0$ and 5.0,1H), $2.43(\mathrm{~m}, 1 \mathrm{H}), 2.36(\mathrm{~m}$, 1H), $2.23(\mathrm{~m}, 1 \mathrm{H}), 2.13(\mathrm{~m}, 1 \mathrm{H}), 1.97(\mathrm{~m}, 1 \mathrm{H}), 1.45(\mathrm{~s}, 9 \mathrm{H}), 1.44(\mathrm{~s}, 9 \mathrm{H}), 1.28(\mathrm{~s}, 9 \mathrm{H})$ $1.08(\mathrm{~d}, \mathrm{~J}=6.5,3 \mathrm{H}), 0.98(\mathrm{~d}, \mathrm{~J}=8.0,3 \mathrm{H}), 0.96(\mathrm{~d}, \mathrm{~J}=7.0,3 \mathrm{H})$.

$H-C(T r t) T(T B u) E(t B u) V D(t B u)-O M e$. Z-T(tBu)E(tBu)VD(tBu)-OMe was deprotected and coupled to Fmoc-C(Trt)-OH. The Fmoc protecting group was then removed to yield $0.470 \mathrm{~g}(92 \%)$ of $\mathrm{H}-\mathrm{C}(\mathrm{Trt}) \mathrm{T}(\mathrm{TBu}) \mathrm{E}(\mathrm{tBu}) \mathrm{VD}(\mathrm{tBu})-\mathrm{OMe} .{ }^{1} \mathrm{H}-\mathrm{NMR}$ $(500 \mathrm{MHz}): \delta 7.81(\mathrm{~d}, \mathrm{~J}=6.3,1 \mathrm{H}), 7.69(\mathrm{~d}, \mathrm{~J}=7.4,1 \mathrm{H}), 7.45(\mathrm{~m}, 6 \mathrm{H}), 7.23(\mathrm{~m}, 9 \mathrm{H}), 6.99$ $(\mathrm{d}, \mathrm{J}=8.4,1 \mathrm{H}), 6.90(\mathrm{~d}, \mathrm{~J}=8.4,1 \mathrm{H}), 4.81(\mathrm{dt}, \mathrm{J}=8.9$ and 4.7, $1 \mathrm{H}), 4.37(\mathrm{dd}, \mathrm{J}=12.9$ and 7.7, 1H), $4.23(\mathrm{~m}, 2 \mathrm{H}), 4.11(\mathrm{~m}, 1 \mathrm{H}), 3.71(\mathrm{~s}, 3 \mathrm{H}), 2.90(\mathrm{~m}, 2 \mathrm{H}), 2.70(\mathrm{~m}, 2 \mathrm{H}), 2.34(\mathrm{~m}$, 
4H), $2.24(\mathrm{~m}, 2 \mathrm{H}), 1.93(\mathrm{~m}, 1 \mathrm{H}), 1.42(\mathrm{~s}, 18 \mathrm{H}), 1.24(\mathrm{~s}, 9 \mathrm{H}), 0.97(\mathrm{~d}, \mathrm{~J}=6.6,3 \mathrm{H}), 0.95(\mathrm{~d}$, $\mathrm{J}=6.7,3 \mathrm{H})$.

Norbornene-C(Trt)T(tBu)E(tBu)VD(tBu)-OMe $3 \boldsymbol{b}$. H-C(Trt)T(TBu)E(tBu)VD(tBu)OMe was coupled to 5-norbornene-exo-carboxylic acid to yield $0.400 \mathrm{~g}(90 \%)$ of norbornene-C(Trt)T(tBu)E(tBu)VD(tBu)-OMe, 3b. ${ }^{1} \mathrm{H}-\mathrm{NMR}(600 \mathrm{MHz}): \delta 7.42$ (m, 6H), $7.30(\mathrm{~m}, 6 \mathrm{H}), 7.25(\mathrm{~m}, 4 \mathrm{H}), 6.92(\mathrm{~m}, 2 \mathrm{H}), 6.89(\mathrm{~d}, \mathrm{~J}=6.6,1 \mathrm{H}), 6.13(\mathrm{~m}, 2 \mathrm{H}), 5.67(\mathrm{~d}$, $\mathrm{J}=4.8,1 \mathrm{H}), 4.82(\mathrm{~m}, 1 \mathrm{H}), 4.37(\mathrm{~m}, 1 \mathrm{H}), 4.27(\mathrm{~m}, 2 \mathrm{H}), 4.13(\mathrm{~m}, 1 \mathrm{H}), 4.00(\mathrm{~m}, 1 \mathrm{H}), 3.72$ (s, 3H), 2.87(m, 2H), $2.76(\mathrm{~m}, 2 \mathrm{H}), 2.66(\mathrm{~m}, 1 \mathrm{H}), 2.33(\mathrm{~m}, 3 \mathrm{H}), 2.14(\mathrm{~m}, 1 \mathrm{H}), 1.92(\mathrm{~m}$, 2H), $1.83(\mathrm{~d}, \mathrm{~J}=12.0$ and 3.3, 1H), $1.43(\mathrm{~s}, 9 \mathrm{H}), 1.42(\mathrm{~s}, 9 \mathrm{H}), 1.27(\mathrm{~m}, 2 \mathrm{H}), 1.17(\mathrm{~s}, 9 \mathrm{H})$, $1.07(\mathrm{~d}, \mathrm{~J}=6.6,3 \mathrm{H}), 0.95(\mathrm{~d}, \mathrm{~J}=6.6,3 \mathrm{H}), 0.93(\mathrm{~d}, \mathrm{~J}=6.6,3 \mathrm{H}) .{ }^{13} \mathrm{C}(250 \mathrm{MHz}) \delta 17.68$, $19.05,19.87,26.11,26.51,27.27,27.99,28.44,30.45,30.67,31.90,33.96,37.18,38.54$, 41.54, 44.39, 46.21, 47.06, 47.67, 48.53, 49.26, 51.02, 52.47, 52.59, 58.55, 65.39, 66.67, $67.65,68.05,74.32,80.77,81.73,126.79,127.92,129.39,135.84,138.24,144.23,169.98$, $170.44,170.59,170.98,171.12,172.58,175.02,175.05$. MALDI, calcd for $(\mathrm{MNa})^{+} 1132.89$, found 1133.96 . HPLC purity was $96 \%$.

\section{Bicyclo[2.2.1] hept-5-ene-exo-2-carboxylic acid norbornyl $N$-hydroxysuccinimide}

ester, 2. The N-hydroxysuccinimide ester 2 was prepared by the procedure of Strong and Kiessling with minor modifications. ${ }^{2}$ The norbornene carboxylic acid (500 mg, $3.6 \mathrm{mmol}$ ) was activated with TBTU (1.0 eq) and the $N$-hydroxy ester $(463 \mathrm{mg}, 4.0 \mathrm{mmol})$ was added to the mixture. The reaction was stirred in $\mathrm{CH}_{2} \mathrm{Cl}_{2}(5 \mathrm{~mL})$ at $\mathrm{RT}$ for $6 \mathrm{~h}$. The solvent was removed and the residue was subjected to flash silica gel chromatography

\footnotetext{
${ }^{2}$ Strong, L. E.; Kiessling, L. L. J. Am. Chem. Soc. 1999, 121, 6193-6196.
} 
with $\mathrm{CH}_{2} \mathrm{Cl}_{2}$ as the solvent. A white solid was isolated. Yield $714 \mathrm{mg}(84 \%){ }^{1} \mathrm{H}-\mathrm{NMR}$ $\left(\mathrm{CDCl}_{3}, 300 \mathrm{MHz}\right) \delta 6.23(\mathrm{dd}, \mathrm{J}=5.9,2.9 \mathrm{~Hz}, 1 \mathrm{H}), 6.17(\mathrm{dd}, \mathrm{J}=5.7,3.0 \mathrm{~Hz}, 1 \mathrm{H}), 3.30$ (br s, 1H), 3.00 (br s, 1H), 2.87 (d, J = 2.1 Hz, 4H), 2.54 (ddd, J = 8.9, 4.4, 1.4 Hz, 1H), $2.08(\mathrm{dt}, \mathrm{J}=12.2,4.1 \mathrm{~Hz}, 1 \mathrm{H}), 1.44-1.62(\mathrm{~m}, 3 \mathrm{H}) .{ }^{13} \mathrm{C}(400, \mathrm{MHz}) \delta 25.84,31.21$, 40.54, 42.01, 46.65, 47.36, 135.51, 138.78, 169.48, 171.86. HPLC purity was $97 \%$.

\section{Polymerization}

Typical polymerization procedure. Catalyst $\mathrm{Cl}_{2}\left(\mathrm{PCy}_{3}\right)\left(\mathrm{H}_{2} \mathrm{IMes}\right) \mathrm{Ru}=\mathrm{CHPh}(26 \mathrm{mg}$, $0.30 \mathrm{mmol}$ ) was weighed in an $\mathrm{N}_{2}$-filled drybox and dissolved in $1 \mathrm{~mL} \mathrm{CH}_{2} \mathrm{Cl}_{2} / \mathrm{CH}_{3} \mathrm{OH}$ (3/1) to give a concentration of $0.03 \mathrm{M}$. An aliquot of the catalyst $(16.6 \mu \mathrm{L}, 0.005 \mathrm{mmol})$ was added to $2(1.175 \mathrm{mg}, 0.05 \mathrm{mmol})$ (1:1 molar ratio) in a reaction bottle under an inert Ar atmosphere. The reaction was stirred at RT for $2 \mathrm{~h}$. Peptide monomer $(0.05$ mmol 3a or 3b) was dissolved in $50 \mu \mathrm{L}$ of $\mathrm{CH}_{2} \mathrm{Cl}_{2} / \mathrm{CH}_{3} \mathrm{OH}(3 / 1)$. This solution was added to the reaction mixture and the mixture stirred for 3 to $4 \mathrm{~h}$ before quenching with $20 \mu \mathrm{L}$ of ethyl vinyl ether and stirring for an additional $30 \mathrm{~min}$. The solvent was concentrated and the product was precipitated with cold $\mathrm{Et}_{2} \mathrm{O}$. Product was isolated by centrifugation and dried under vacuum in the presence of $\mathrm{P}_{2} \mathrm{O}_{5}$.

The product polymer (30 mg, $0.0043 \mathrm{mmol}$ ) was deprotected in $2 \mathrm{~mL}$ of cocktail containing $\mathrm{H}_{2} \mathrm{O}$, TIS and TFA (2.5/2.5/95) for $4 \mathrm{~h}$. The reaction mixture was concentrated to $100 \mu \mathrm{L}$ with $\mathrm{N}_{2}$ and precipitated with cold $\mathrm{Et}_{2} \mathrm{O}$ and the solid $4 \mathbf{a}$ or $4 \mathbf{b}$ collected by centrifugation.

Typical fluorophore coupling procedure. Polymer 4a or 4b (10 mg, $0.0014 \mathrm{mmol})$ was dissolved in $30 \mu \mathrm{L}$ dried DMF and Oregon Green 488 cadaverine $(3 \mathrm{mg}, 0.006$ 
mmol) dissolved in $30 \mu \mathrm{L}$ of dried DMF, was added to the polymer solution followed by $28 \mu \mathrm{L}$ of DIPEA $(0.16 \mu \mathrm{mol})$. The conjugation was allowed to proceed for $2 \mathrm{~h}$ under Ar. The reaction mixture was mixed with $800 \mu \mathrm{L} \mathrm{H}_{2} \mathrm{O}$, concentrated to $50 \mu \mathrm{L}$ by evaporation, and the product was precipitated with cold $\mathrm{Et}_{2} \mathrm{O}$. Product $\mathbf{5 a}$ or $\mathbf{5 b}$ was isolated by centrifugation and dried under vacuum in the presence of $\mathrm{P}_{2} \mathrm{O}_{5}$.

Polymer 5a or 5b ( $8 \mathrm{mg}, 0.0001 \mathrm{mmol})$ was dissolved in $200 \mu \mathrm{L} \mathrm{H}_{2} \mathrm{O}$ at $\mathrm{pH} 6$ and reduced with $20 \mathrm{mM}$ TCEP for $2 \mathrm{~h}$ with stirring at RT. The reduced product was isolated by precipitation with $500 \mu \mathrm{L} 1 \mathrm{~N} \mathrm{HCl}$. Excess TCEP was removed by washing with $3 \mathrm{X} 100 \mu \mathrm{L} \mathrm{H}_{2} \mathrm{O}$. The reduced $\mathbf{5 a}$ or $\mathbf{5 b}$, a light yellow solid, was collected, dried and stored at $-20{ }^{\circ} \mathrm{C}$.

Polymer 4a.Yield $70 \mathrm{mg}(83 \%){ }^{1} \mathrm{H}-\mathrm{NMR}$ (DMSO, $\left.600 \mathrm{MHz}\right) \delta 8.45-7.00$ (with max. at $8.24,7.80,7.45,7.23,7.15$ ), 5.24 (br s), $4.33-4.00$ (with max. at 4.54, 4.33, 4.08), $3.56(\mathrm{~s}), 3.29(\mathrm{~s}), 3.20-2.23$ (with max. at 2.74, 2.61, 2.48, 2.35), $2.20-1.45$ (with max. at 2.11, 1.88, 1.79, 1.64), $1.26(\mathrm{br} \mathrm{s}), 1.08(\mathrm{~m}), 0.68(\mathrm{~m})$. GFC purity was $98 \%$. Polymer 4b.Yield $57 \mathrm{mg}\left(78 \%\right.$ ) ${ }^{1} \mathrm{H}-\mathrm{NMR}$ (DMSO, $\left.500 \mathrm{MHz}\right) \delta 8.62-7.60$ (with max. at $8.40,8.05,7.89), 5.20(\mathrm{~m}), 4.76-3.80$ (with max. at 4.50, 4.36, 4.20, 4.00), 3.10 -2.40 (with max. at $2.80,2.67,2.69,2.50$ ), $2.30-1.35$ (with max. at $2.29,1.85,1.70$ ), $1.30-0.60$ (with max at $1.22,1.16,1.00,0.85$ ). GFC purity was $98 \%$.

Polymer 5a.Yield $16 \mathrm{mg}(80 \%){ }^{1} \mathrm{H}-\mathrm{NMR}\left(\mathrm{D}_{2} \mathrm{O}, 600 \mathrm{MHz}\right) \delta 7.20(\mathrm{~m}), 5.30(\mathrm{br} \mathrm{s})$, $4.21(\mathrm{~m}), 3.22$ (br s), $3.14-2.30$ (with max. at 2.71, 2.56), $2.29-1.55$ (with max. at 2.12, 1.88), $1.23-1.02$ (with max. at 1.19, 1.07), $0.84(\mathrm{~m})$. Expected integration for $\delta$ 7.20/83.22: 6/30; observed: 5/35. GFC purity was $96 \%$. 
Polymer 5b.Yield $53 \mathrm{mg}(74 \%){ }^{1} \mathrm{H}-\mathrm{NMR}\left(\mathrm{D}_{2} \mathrm{O}, 600 \mathrm{MHz}\right) \delta 7.25(\mathrm{~m}), 5.29$ (br s), $4.18(\mathrm{~m}), 3.24$ (s), $3.25-2.28$ (with max. at 2.65, 2.52, 2.49, 2.42), $2.24-1.40$ (with max. at 2.32, 2.00, 1.94, 1.82), $1.08(\mathrm{~m}), 0.80(\mathrm{~m})$. Expected integration for $\delta$ 7.25/83.24: 5/30; observed: 5/48. GFC purity was $97 \%$. 
Norbonyl Succinimide ester

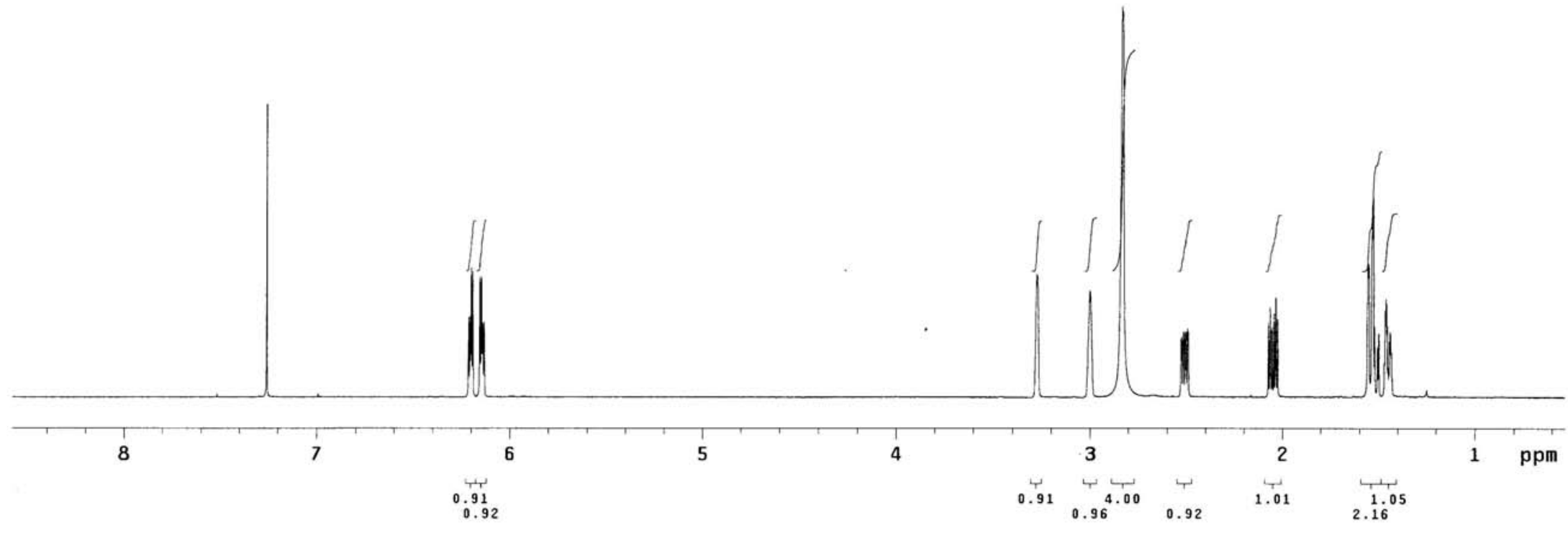


Norbornyl succinimide ester

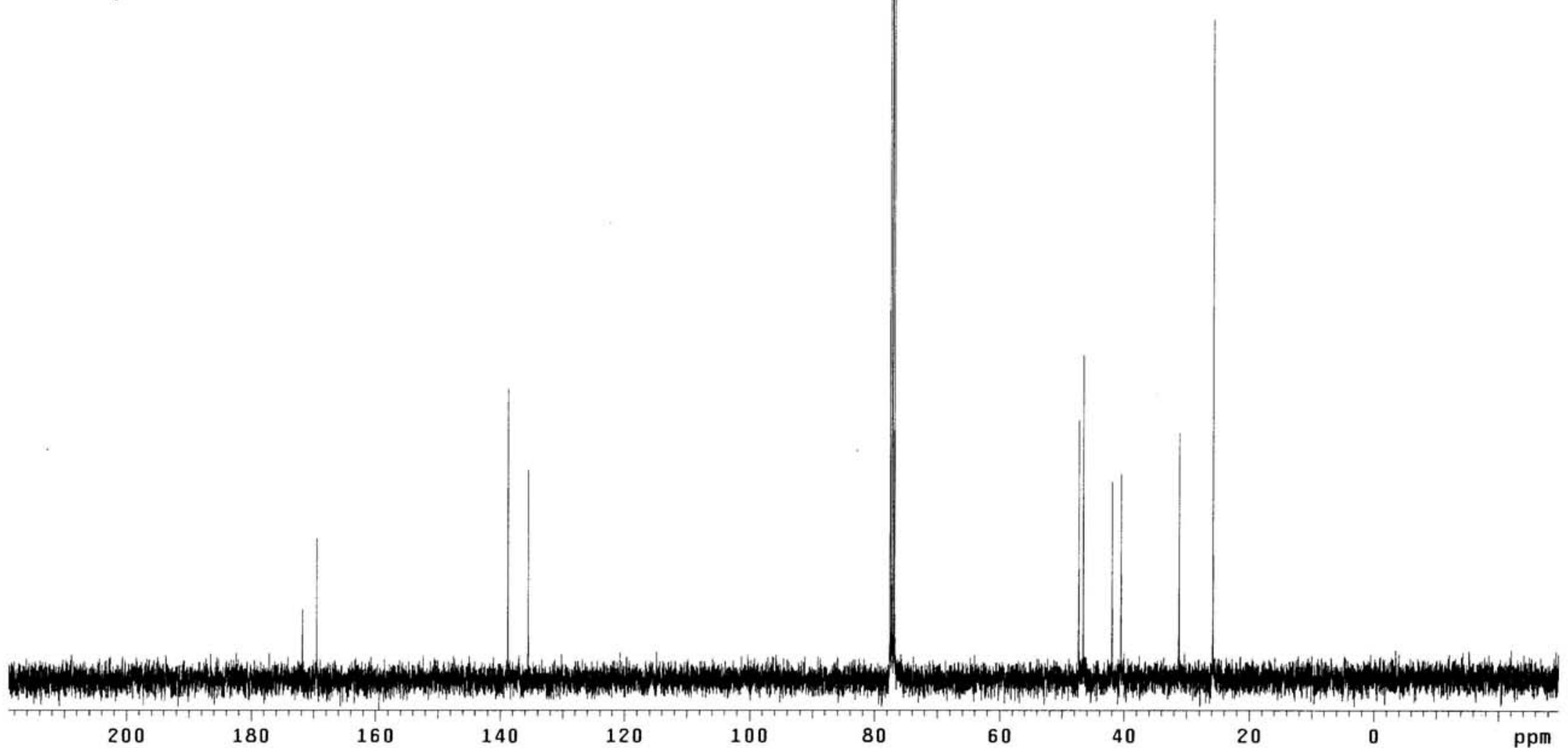




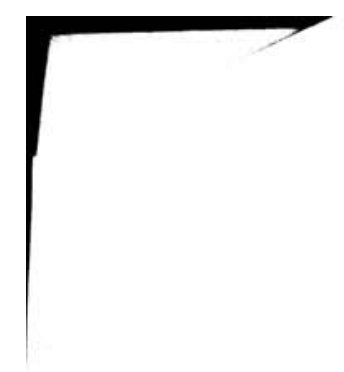

Polymer 4a

$\mathrm{n}=10$

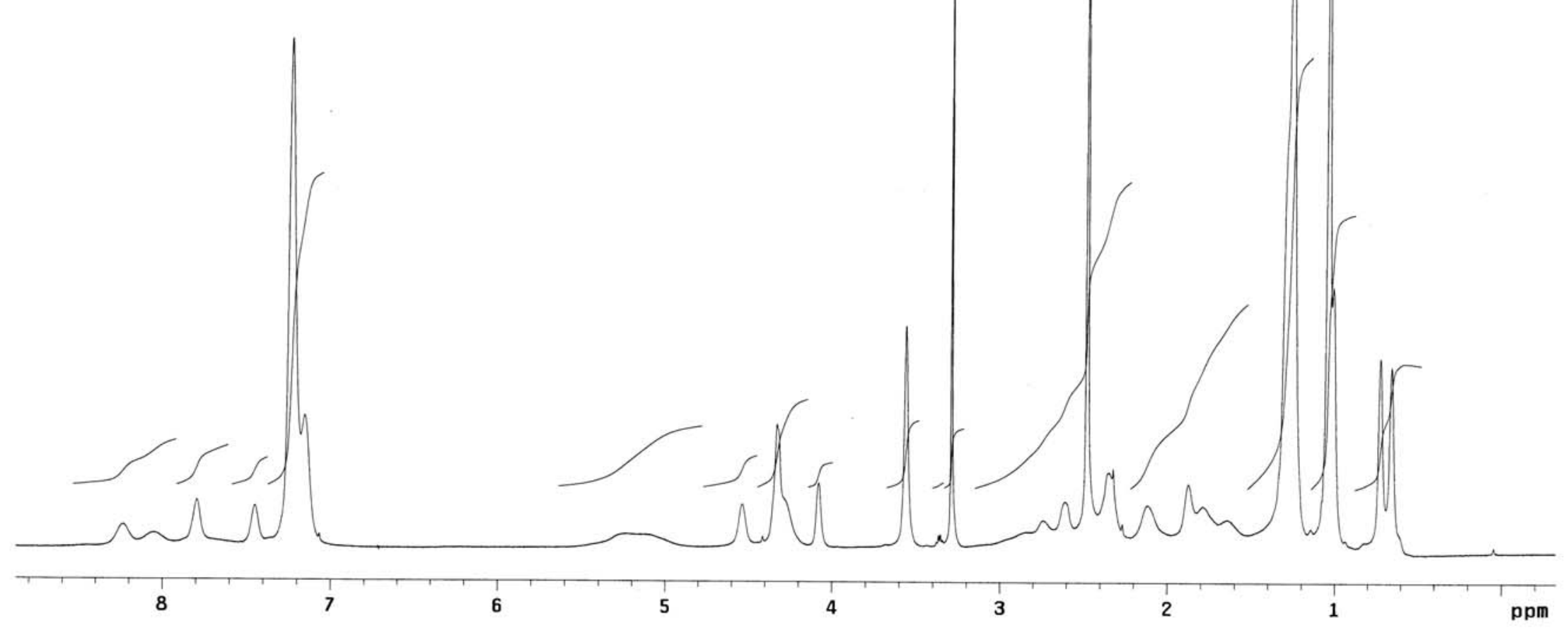




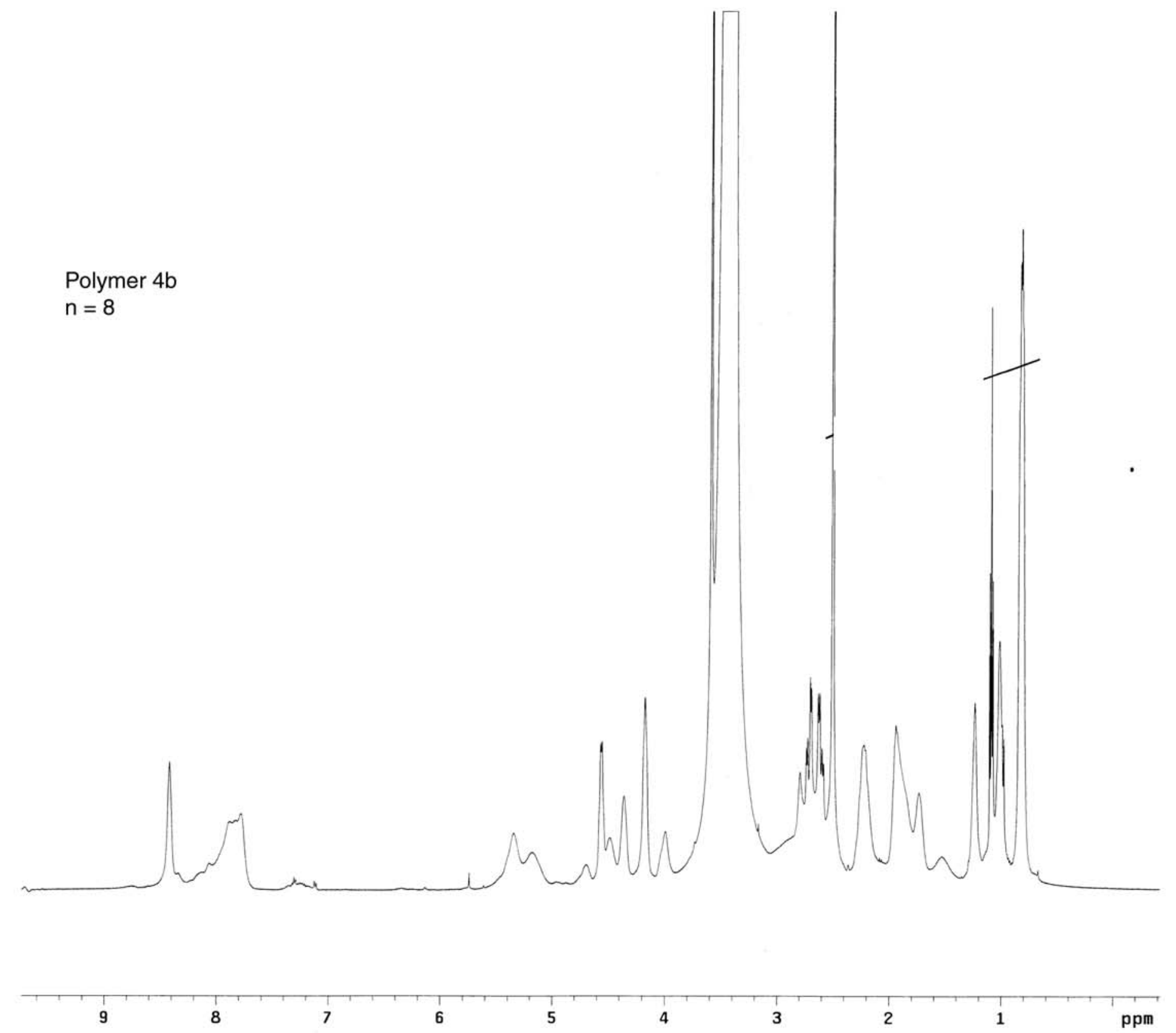


Polymer 5a

$\mathrm{n}=10$

Fluo ECD vit Pdyme

$\mathrm{BXN}_{3}$

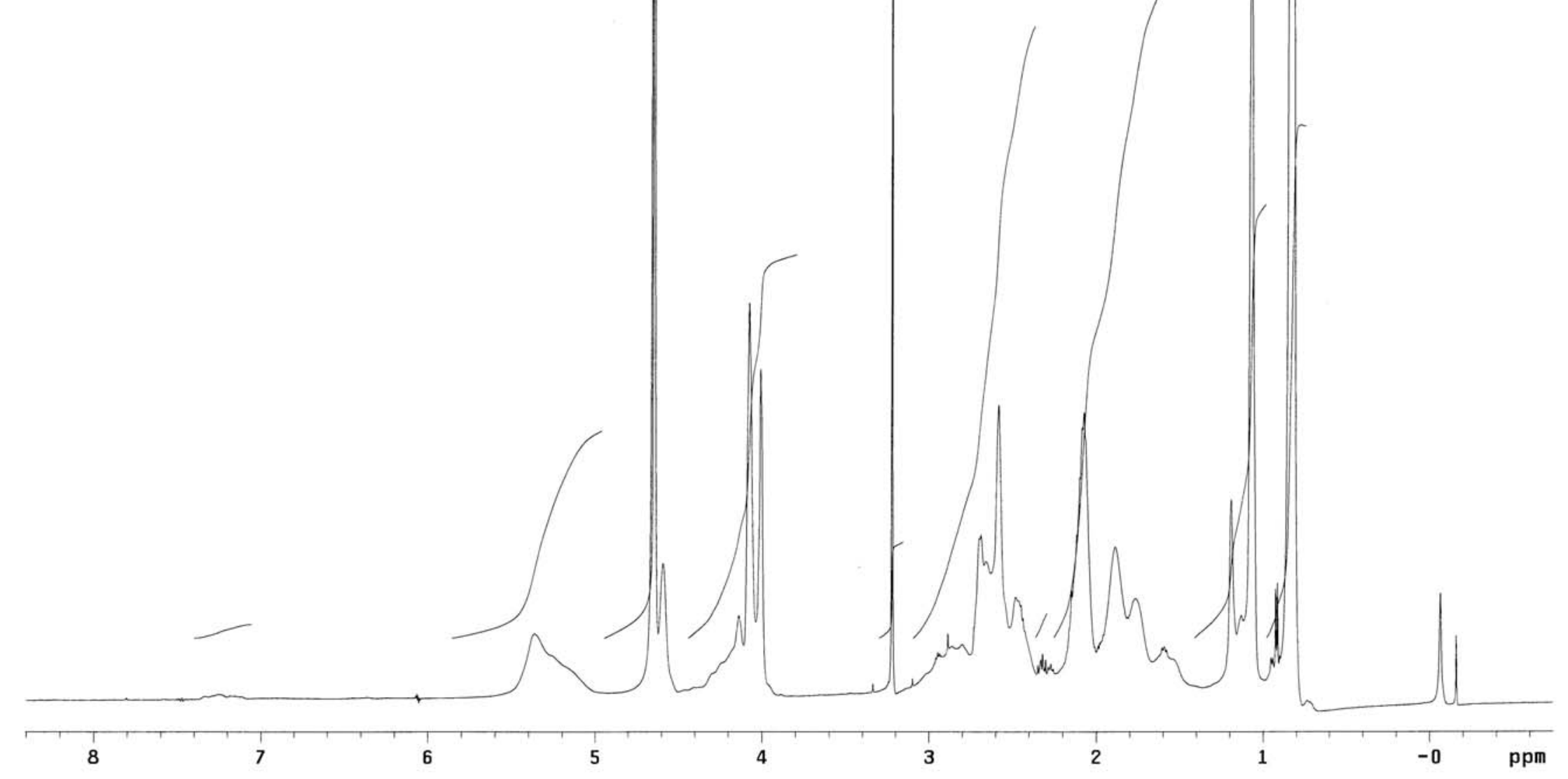


Polymer 5b

$n=8$

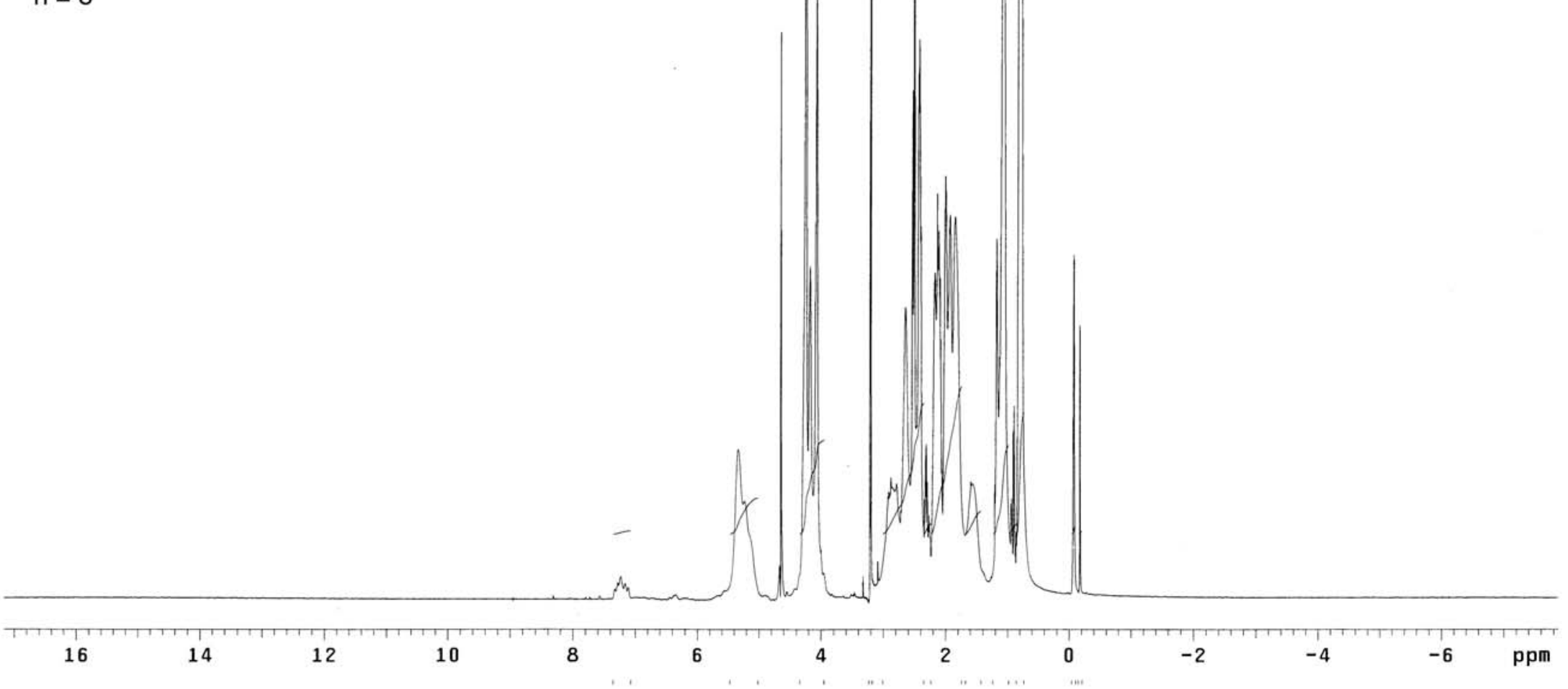

